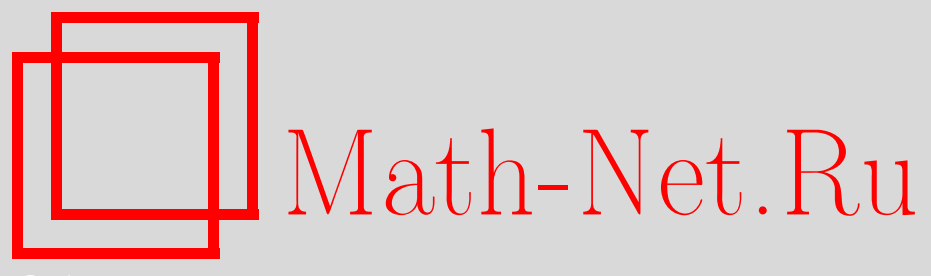

С. Ф. Чичоян, О гладкости решений задачи Дирихле для бигармонического уравнения в негладких двумерных областях, Матем. заметки, 2015, том 98, выпуск 6, 952954

DOI: https://doi.org/10.4213/mzm10978

Использование Общероссийского математического портала Math-Net.Ru подразумевает, что вы прочитали и согласны с пользовательским соглашением http://www.mathnet.ru/rus/agreement

Параметры загрузки:

IP : 54.174 .149 .18

26 апреля 2023 г., 03:55:40

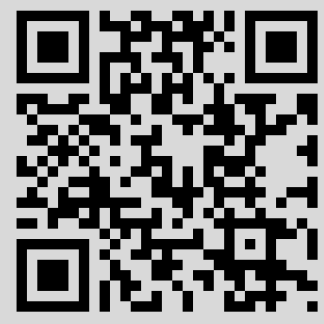




\section{О гладкости решений задачи Дирихле для бигармонического уравнения в негладких двумерных областях}

\section{С. Ф. Чичоян}

Пусть $\Omega$ - ограниченная область в $\mathbb{R}^{2}$, граница $\partial \Omega$ которой содержит точку 0 - начало координат, $\bar{\Omega}=\Omega \cup \partial \Omega$ - замыкание $\Omega, x=\left(x_{1}, x_{2}\right),|x|=\sqrt{x_{1}^{2}+x_{2}^{2}}$.

В области $\Omega$ рассматривается задача Дирихле для бигармонического уравнения

$$
\Delta^{2} u(x)=f(x), \quad f(x) \in L^{2}(\Omega),
$$

с граничными условиями

$$
\left.u(x)\right|_{\partial \Omega}=0,\left.\quad \operatorname{grad} u(x)\right|_{\partial \Omega}=0 .
$$

Вопросы об асимптотике, гладкости, единственности и методы их исследования подобных задач развиты в работах Кондратьева, Олейник и их учеников, [1]-[9]. В частности, в работах [1]-[5] для решения задачи Дирихле для бигармонического уравнения получены оценки в окрестности нерегулярных точек границы при слабых предположениях относительно структуры границы в окрестности этих точек, а также оценки, характеризующие поведение решений в окрестности нерегулярных точек границы. Изучено поведение обобщенного решения задачи Дирихле для бигармонического уравнения в окрестности граничной точки и в окрестности бесконечности в случае двух независимых переменных. Кроме того, получены энергетические оценки, выражающие принцип Сен-Венана в теории упругости для произвольного плоского тела, доказаны теоремы единственности решения задачи Дирихле для неограниченных областей в классе функций, зависящем от геометрии области. Также получены неулучшаемые оценки характеризующие поведение $|u(x)|$ и $|\nabla u(x)|$ при $|x| \rightarrow \infty$.

В работе [6] с условием конечности интеграла Дирихле на поведение решения на бесконечности изучены вопросы единственности решений краевых задач для эллиптических систем высокого порядка в неограниченных областях. В разных классах неограниченных областей с конечным весовым интегралом энергии (Дирихле) изучены вопросы единственности и найдены размерности пространства решений краевых задач для системы теории упругости и бигармонического (полигармонического) уравнения в работах [7]-[9].

Поведение при $|x| \rightarrow \infty$ обобщенных решений задачи Дирихле для системы уравнений Навье-Стокса и системы Кармана в окрестности бесконечно удаленной точки рассматривались в работе [10], а гладкость обобщенных решений задачи Дирихле для системы уравнений Навье-Стокса в негладких двумерных областях в [11], в которых при определенных условиях геометрического характера на границу области получены также оценки, характеризующие поведение решений задачи Дирихле.

В данной заметке методом весовых функций, изучаются вопросы гладкости решений задачи Дирихле для бигармонического уравнения в двумерной области с негладкой границей.

Введем следующие обозначения:

- $C_{0}^{\infty}(\Omega)$ - пространство бесконечно диффернцируемых функций в области $\Omega$, имеющих компактный носитель в $\Omega$;

- $H^{k}(\Omega)$ - пространство Соболева, полученное пополнением $C^{\infty}(\bar{\Omega})$ по норме

$$
\|u(x)\|_{H^{k}(\Omega)}^{2}=\int_{\Omega} \sum_{|\alpha| \leqslant k}\left|D^{\alpha} u(x)\right|^{2} d x,
$$

где $D^{\alpha} \equiv \partial^{|\alpha|} /\left(\partial x_{1}^{\alpha_{1}} \partial x_{2}^{\alpha_{2}}\right), \alpha=\left(\alpha_{1}, \alpha_{2}\right)$ - мультииндекс, $\alpha_{j}$ - целые неотрицательные числа, $|\alpha|=\alpha_{1}+\alpha_{2}, k \in \mathbb{N}$;

DOI: $10.4213 / \mathrm{mzm} 10978$

(C) С.Ф. Чичоян, 2015 
- $\stackrel{\circ}{H}^{k}(\Omega)$ - пространство функций в $\Omega$, полученное пополнением $C_{0}^{\infty}(\Omega)$ по норме пространства Соболева $H^{k}(\Omega)$.

Положим

$$
\begin{gathered}
E(u, v)=\sum_{|\alpha|=2} \frac{2}{\alpha !} D^{\alpha} u D^{\alpha} v, \quad E(u)=E(u, u), \quad \alpha !=\alpha_{1} ! \alpha_{2} !, \\
M_{\Omega}(u)=\inf _{Q}\left(\frac{1}{\operatorname{mes} Q} \int_{\Omega} u^{2}(x) d x\right)^{1 / 2},
\end{gathered}
$$

где $Q$ - любое ограниченное множество в $\Omega$.

ОПредЕлЕниЕ 1. Обобщенным решением задачи (1), (2) назовем функцию $u(x) \in \stackrel{\circ}{H}^{2}(\Omega)$, для которой при любой функции $v(x) \in \stackrel{\circ}{H}^{2}(\Omega)$ выполнено интегральное тождество

$$
\int_{\Omega} E(u, v) d x=\int_{\Omega} f(x) v(x) d x .
$$

Установим следующие вспомогательные утверждения.

Лемма 1. Пусть $и(x) \in H^{1}(G)$. Тогда найдется постоянная $C$, не зависящая от $и(x)$, такая, что

$$
\|u(x)\|_{H^{1}(G)} \leqslant C\left(\left(\int_{G}|\nabla u|^{2} d x\right)^{1 / 2}+M_{G}(u)\right) .
$$

ДокАзАтельство. Предположим противное. Тогда для всякого натурального $n$ должно существовать $u_{n} \in H^{1}(G)$ такое, что

$$
\left\|u_{n}\right\|_{H^{1}(G)}>n\left(\left(\int_{G}\left|\nabla u_{n}\right|^{2} d x\right)^{1 / 2}+M_{G}\left(u_{n}\right)\right) .
$$

Обозначая $v_{n}=u_{n} /\left\|u_{n}\right\|_{H^{1}(G)}$, будем, очевидно, иметь

$$
\begin{gathered}
\left\|v_{n}\right\|_{H^{2}(G)}=1, \quad n=1,2, \ldots \\
\left(\int_{G}\left|\nabla u_{n}\right|^{2} d x\right)^{1 / 2}+M_{G}\left(u_{n}\right) \leqslant \frac{1}{n}, \quad n=1,2, \ldots .
\end{gathered}
$$

Последовательность $v_{n}, n=1,2, \ldots$, ограничена в $H^{1}(G)$, поэтому из нее можно выделить подпоследовательность, фундаментальную в $L^{2}(G)$. Для простоты будем обозначать эту подпоследовательность также $v_{n}, n=1,2, \ldots$. Из (4) следует, что эта последовательность будет фундаментальна и в $H^{1}(G)$, а значит,

$$
\lim _{n \rightarrow \infty}\left\|v_{n}-v\right\|_{H^{1}(G)}=0 \quad \text { для некоторого } \quad v \in H^{1}(G) .
$$

Причем, $|\nabla v|=0$. Таким образом, $v(x)=$ const. Из (4) имеем $M_{G}(v)=v(x)=0$. Тем самым, мы приходим к противоречию с (3). Лемма доказана.

Лемма 2. Пусть $u(x) \in H^{2}\left(\theta_{\rho}\right)$, где $\theta_{\rho}=\{x: \rho<|x|<2 \rho\}$ и в $\theta_{\rho}$

$$
M_{\theta_{\rho}}(u)=M_{\theta_{\rho}}\left(u_{x_{i}}\right)=0, \quad i=1,2 .
$$

Тогда

$$
\int_{\theta_{\rho}} \rho^{-4} u^{2} d x+\int_{\theta_{\rho}} \rho^{-2}|\nabla u|^{2} d x \leqslant C \int_{\theta_{\rho}} \sum_{|\alpha|=2}\left|D^{\alpha} u\right|^{2} d x,
$$

где постоянная $C$ не зависит от $и$ и $\rho$.

ЗАмечАниЕ 1 . Лемма 2 получается из леммы 1 с помощью растяжения области. 
Основным результатом этой заметки является следующая теорема.

Теорема 1. Пусть функиия $u(x) \in \stackrel{\circ}{H}^{2}(\Omega)$ является обобщенным решением задачи Дирихле (1), (2), $f(x) \in L^{2}(\Omega)$. Пусть начало координат $0 \in \partial \Omega$, и в каждом кольие $\theta^{k}=\left\{x: 2^{-k}<|x|<2^{-k+1}\right\}$ nрu $k \geqslant k_{0}$

$$
M_{\theta^{k}}(u)=M_{\theta^{k}}\left(u_{x_{i}}\right)=0, \quad i=1,2 .
$$

Тогда существует постоянная $\beta>0$, зависящая от $f(x)$ u $\Omega$, такая, что

$$
\begin{gathered}
\int_{\Omega}|x|^{-\beta} \sum_{|\alpha|=2}\left|D^{\alpha} u\right|^{2} d x+\int_{\Omega}|x|^{-\beta-2}|\nabla u|^{2} d x+\int_{\Omega}|x|^{-\beta-4} u^{2}(x) d x \leqslant C \int_{\Omega} f^{2}(x) d x, \\
|u(x)|^{2} \leqslant C|x|^{2+\beta} \int_{\Omega} f^{2}(x) d x
\end{gathered}
$$

где постоянная $C>0$ не зависит от $и$.

ДокАЗАТЕльство теоремы основано на леммах 1, 2 и теоремах вложения. Для получения оценок (5), (6) в интегральном тождестве вводится весовая пробная функция вида $v(x)=u(x)|x|^{-\beta}, \beta=$ const $>0$, и устанавливается оценка для модуля решения в окрестности нерегулярной граничной точки.

\section{СПИСОК ЦИТИРОВАННОЙ ЛИТЕРАТУРЫ}

[1] О.А. Олейник, Г.А. Иосифьян, Сиб. матем. журн., 19:5 (1978), 1154-1165. [2] О. А. Олейник, Г. А. Иосифьян, И. Н. Тавхелидзе, Тр. ММО, 42, Изд-во Моск. ун-та, М., 1981, 160-175. [3] О. А. Олейник, В. А. Кондратьев, И. Копачек, Дифферени. уравнения, 17:10 (1981), 1886-1899. [4] V. A. Kondratiev, O. A. Oleinik, Proc. Roy. Soc. Edinburgh Sect. A, 93 (1983), 327-343. [5] В. А. Кондратьев, И. Копачек, Д. М. Леквеишвили, О. А. Олейник, Современные проблемы математики. Дифференциальные уравнения, математический анализ и их приложения, Тр. МИАН СССР, 166, 1984, 91-106. [6] А. А. Коньков, Матем. сб., 184:12 (1993), 23-52. [7] О. А. Матевосян, Дифферени. уравнения, 34:6 (1998), 806-811. [8] О. А. Матевосян, Матем. заметки, 70:3 (2001), 403-418. [9] О. A. Matevosyan, Russ. J. Math. Phys., 21:1 (2014), 130-132. [10] С. Ф. Чичоян, УМH, 41:2 (1986), 211-212. [11] С.Ф. Чичоян, Вестн. Моск. ун-та. Сер. 1. Матем., мех., 1988, № 1, 82-84.

\section{С. Ф. Чичоян}

Московский финансово-юридический университет

E-mail: sargish61@gmail.com
Поступило 02.03.2015 